\title{
Stream Reasoning with LARS
}

\author{
Harald Beck ${ }^{1} \cdot$ Minh Dao-Tran ${ }^{1} \cdot$ Thomas Eiter $^{1} \cdot$ Christian Folie $^{1}$
}

Published online: 17 May 2018

(c) The Author(s) 2018

\begin{abstract}
Stream reasoning is the task of continuously deriving conclusions on streaming data. Different research communities emphasize different aspects such as throughput vs. expressiveness, yet a mathematical model to describe the declarative semantics of such systems has been missing. This motivated the logic-based framework LARS for analytic reasoning over streams. However, it is also attractive for applications by itself.
\end{abstract}

Keywords Stream reasoning - Incremental reasoning

\section{Introduction: Stream Reasoning}

Stream reasoning $[7,10]$ emerged from stream processing [2] to add logic-oriented features on top of processing continuously changing data. The approach of the influential continuous query language (CQL) [1], which can be seen as extension of SQL for streams, is to first obtain windows, i.e., recent snapshots of streaming data, and then execute queries as on a database. This principle has been adopted, e.g., in the Semantic Web area to extend static queries for streams [3, 12]. In Knowledge Representation and Reasoning (KR) some recent works (e.g. $[9,11])$ have addressed incremental reasoning. However, prior to our work, no language extension of Answer Set Programming (ASP; see this issue) for stream reasoning with windows was proposed.

Harald Beck

beck@kr.tuwien.ac.at

Minh Dao-Tran

dao@kr.tuwien.ac.at

Thomas Eiter

eiter@kr.tuwien.ac.at

Christian Folie

christian.folie@outlook.com

1 Institute of Logic and Computation, TU Wien,

Favoritenstraße 9-11, 1040 Vienna, Austria

\section{The LARS Framework}

In contrast to temporal reasoning as in Linear Temporal Logic (LTL), which deals with reasoning about infinite sequences of states, LARS [5] places finite streams and windows at the conceptual core. We model a stream $S$ as a pair $(T, v)$ of a timeline $T$, which is a closed interval in the natural numbers, and an evaluation function $v$ that maps each $t \in T$ to a (possibly empty) set of atoms, i.e., expressions of form $p\left(c_{1}, \ldots, c_{n}\right)$ where $p$ is a predicate and $c_{1}, \ldots, c_{n}$ are constants $(n \geq 0)$. A window function $w$ takes a stream $S$ and a time point $t \in \mathbb{N}$ and returns a substream $w(S, t)$ of $S$ called a window. For instance, consider a stream $S=([0,500], v)$, where $v(485)=v(490)=\{x\}$ and $v(t)=\emptyset$ for all other time points $t$. A (sliding) time-based window of size 10, applied on $S$ at time 500 yields the window $([490,500], v)$, which is also obtained by a tuple-based window of size 1 .

LARS formulas extend propositional logic, in particular by window operators $\boxplus^{w}$, where $w$ is a generic window function. A formula $\varphi$ is evaluated on a stream $S$ at a time $t$, where $\boxplus^{w} \varphi$ means that the evaluation of $\varphi$ is restricted to the window $w(S, t)$. In contrast to a CQL-style snapshot semantics that drops timestamps of selected data, LARS offers some control: $\diamond \varphi$ (resp. $\square \varphi$ ) holds if $\varphi$ holds at some (resp. every) time point, and $@_{t} \varphi$ holds if $\varphi$ holds at time $t$ in the window.

LARS programs are sets of rules $\varphi_{0} \leftarrow \varphi_{1}, \ldots, \varphi_{n}$, where each $\varphi_{i}$ is a formula; variables are viewed as schematic placeholder for concrete (ground) values. The following program $P$ describes a simple cooling system that must keep the 
temperature below 7 degrees, where atom $s(V)$ stands for a temperature with value $V$.

$@_{T} h i g h \leftarrow \boxplus^{60 s e c} @_{T} s(V), V>7$

cool $\leftarrow \boxplus^{60 s e c} \square$ high

warn $\leftarrow \boxplus^{\# 5} \diamond s(V), V>12$

Rule (1) abstracts away the specific value: for every second $T$ during the last 60, we associate atom high with $T$ if there is a temperature signal $s(V)$ with $V>7$. Rule (2) then triggers cooling if the temperature has always been high in that interval. Finally, Rule (3) infers a warning if one of the last 5 signal values was above 12 .

Semantically, LARS programs can be seen as extension of ASP for streams, thus carrying over attractive features like minimal model reasoning, nonmonotonicity and recursion. The closed world assumption (as in databases) allows for reasoning based on the absence of data; in general, the stable model semantics then leads to multiple models. This makes LARS applicable for scenarios which require the generation of alternative solutions like in planning, diagnosis or configuration.

Satisfiability and model checking are in ground LARS PSPACE-complete (for tractable window functions), but in practical settings not harder than in ground ASP. In contrast to LTL, ground LARS programs with time windows yield the full class of regular languages; nonground programs can express also intractable languages.

\section{Implementations and Incremental Reasoning}

Besides foundational aspects beyond the scope of this note, we developed Ticker [6], a prototypical stream reasoning engine that comes with two reasoning modes for LARS programs with the above sliding windows. ${ }^{1}$

The first mode repeatedly calls the ASP solver Clingo [8] based on a translation. The second, more efficient mode is incremental; it uses truth maintenance systems to adjust the model of a dynamically updated ASP encoding. In Ticker syntax, the above program $P$ reads:

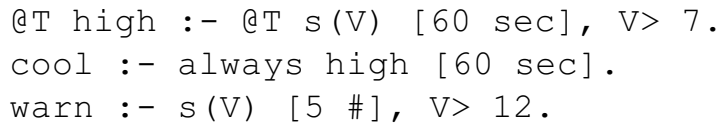

The Laser engine [4] is geared for programs with unique models to achieve high performance, by managing

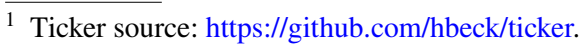

incrementally substitution tables using temporal information. Laser was shown to be significantly faster than C-SPARQL, CQELS or Ticker/Clingo in comparable operations, using Python with PyPy 5.8. ${ }^{2}$

\section{Conclusion}

In conclusion, LARS is a framework that extends ASP for stream reasoning with an emphasis on generic windows. Algorithmically, frequently changing data calls for incremental reasoning, as exemplified in the prototype engines Ticker and Laser. Further and future work includes the use of LARS in applications like network management and dynamic reconfiguration.

Funding Funding was supported by FWF Grants P26471, P27730, and W1255-N23. Open access funding provided by Austrian Science Fund (FWF).

Open Access This article is distributed under the terms of the Creative Commons Attribution 4.0 International License (http://creativeco mmons.org/licenses/by/4.0/), which permits unrestricted use, distribution, and reproduction in any medium, provided you give appropriate credit to the original author(s) and the source, provide a link to the Creative Commons license, and indicate if changes were made.

\section{References}

1. Arasu A, Babu S, Widom J (2006) The CQL continuous query language: semantic foundations and query execution. VLDB J. 15(2):121-142

2. Babu S, Widom J (2001) Continuous queries over data streams. SIGMOD Record 3(30):109-120

3. Barbieri D, Braga D, Ceri S, Della Valle E, Grossniklaus M (2010) C-SPARQL: a continuous query language for RDF data streams. Int J Semantic Comput 4(1):3-25

4. Bazoobandi HR, Beck H, Urbani J (2017) Expressive stream reasoning with Laser. In: Proceeding ISWC

5. Beck H, Dao-Tran M, Eiter T, Fink M (2015) LARS: A logicbased framework for analyzing reasoning over streams. In: Proceeding AAAI, pp 1431-1438. AAAI Press

6. Beck H, Eiter T, Folie C (2017) Ticker: a system for incremental ASP-based stream reasoning. TPLP 17(5-6):744-763

7. Della Valle E, Ceri S, van Harmelen F, Fensel D (2009) It's a streaming world! Reasoning upon rapidly changing information. IEEE Intell Syst 24:83-89

8. Gebser M, Kaminski R, Kaufmann B, Schaub T (2014) Clingo $=\mathrm{ASP}+$ control: Preliminary report. TPLP, online supplement; also CoRR, abs/1405.3694

9. Gebser M, Kaminski R, Obermeier P, Schaub T (2015) Ricochet robots reloaded: A case-study in multi-shot ASP solving. In: LNCS 9060, Springer, pp. 17-32

10. Mileo A, Dao-Tran M, Eiter T, Fink M (2018) Stream reasoning. In: Liu L, Özsu MT (eds) Encyclopedia of database systems, 2nd (edn), Springer, New York (ISBN 978-1-4614-8266-6)

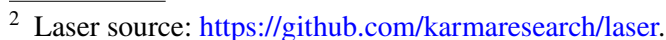


11. Motik B, Nenov Y, Piro R, Horrocks I (2015) Incremental update of datalog materialisation: the backward/forward algorithm. In: Proceeding AAAI, AAAI Press , pp 1560-1568
12. Phuoc DL, Dao-Tran M, Parreira JX, Hauswirth M (2011) A native and adaptive approach for unified processing of linked streams and linked data. In: Proceeding ISWC 